\title{
Objective Transmission Gratings for Large Schmidt Telescopes
}

\author{
John Strong and Fritz Zwicky
}

\begin{abstract}
Several lamellar gratings of 18 -in, aperture, with 300 diffraction elements per inch, have been made for determining stellar spectra with wide angle telescopes. Central orders are missing at $\lambda 4800$, and weak at adjacent wavelengths. The two, equal first orders are about $1^{m}$ weaker than spectra by a prism or echelette grating. Dispersion is linear. The procedure of manufacture is adaptable to larger size. The wavelength at which the central order is missing can be controlled by varying the lamellae thickness. The lamellae are evaporated strips of quartz of $0.5-\mu$ thickness, covering half the face of the support-a spectacle crown, plane parallel plate of $\frac{3}{8}$-in. thickness. The lamellae were deposited by thermal evaporation.
\end{abstract}

In 1940, Zwicky assembled a mosaic of six transparent grating replicas (each 5 in. $\times 7$ in.). He tested the mosaic with the 18-in. Schmidt telescope. The replicas were supplied by R. W. Wood. They were so effectively blazed that the incident light was almost all thrown into one of the two first order spectra. Other stellar spectra and central star images were very weak.

Following the successful test of this grating, Wood and Zwicky made two mosaic gratings, each large enough to cover the 18 -in. Schmidt aperture. One mosaic had 800 lines/in.; the other 1440 lines/in. In contrast with objective prisms that had been prepared (in 1937) for the 18-in. Schmidt telescope, the grating has two desirable features: a linear dispersion and a fiducial mark for wavelength determinations, provided by the weak central image. Nova Cygni of 1942 was discovered with one of these mosaic gratings; and several regions of the sky were surveyed successfully for special objects. This included a survey of $B$ emissionline stars in the double cluster Perseus.

These mosaic gratings had prism-faceted groovesthe prism angle being such that incident rays were refracted at the angle of a first order spectrum. The single slit diffraction pattern of a groove envelops the intensity distribution of the grating. Since the first minima of this diffraction pattern coincides with the angles of the zero and second grating orders, these orders are weak or missing. Hence, with adjacent orders thus forbidden, the blazed first order spectrum of a Wood-type grating was bright.

In 1955, after several vain attempts to devise a practical method of producing large blazed transmission gratings, we turned to the possibilities afforded by

The first author is with the University of Massachusetts, Amherst, Mass; the second, with California Institute of Technology, Pasadena, Calif.

Received 9 October 1968. lamellar type transmission gratings. This type of grating can be fashioned so that it will produce a weak central image with two strong symmetrical first order spectra. Each of these lateral spectra will be about $1^{m}$ less bright than the first order of a Wood-type grating (or of a prism). But, in respect to the Wood grating, the lamellar grating has the advantage of realizability in large size; and in respect to the prism, of linear dispersion. A stellar spectrograph requires a telescope of about $24 \mathrm{in}$. aperture for effective workthe lamellar objective grating may be expected to be equally effective with a telescope of only 16 in. aperture.

Large lamellar transmission gratings are made possible by developments in the art of making halftone screens, such as are used for printing photographs. This art has been highly developed by the Levy Screen Company of Wayne Junction, Pennsylvania.

In 1955, the authors approached the Levy Screen Company and induced them (Edgar B. Coale in particular) to make a 12 -in. $\times 12$-in. lamellar grating with 150 lines/in. It was made on a plane parallel sheet of glass as follows: the plate was coated with a resist which was ruled half away, in strips; the uncovered glass strips were subsequently etched to produce flat-bottomed grooves-each $1 / 300$ in. wide-or 150 parallel grating elements per inch.

Testing the grating with the 18-in. Schmidt, Zwicky showed that this grating produced sharp stellar spectra (at $1800 \AA / \mathrm{mm}$ ) with a dozen Balmer absorption lines clearly distinguished in the spectrum of an A-type star.

Recently, Coale made the 18-in. $\times 18$-in. lamellar type gratings that we report here. Rather than etching, he deposited quartz by thermal evaporation on the resist-removed strips, here $1 / 600$ in. wide, to form flat-topped lands, 300/in. For each grating, a base plate of parallel glass of $\frac{3}{8}$ in. thickness was used. It was water-white spectacle crown glass. Each base plate was given a fine figure by Frank Cooke. 
The new gratings give $926 \AA / \mathrm{mm}$ with the 18 -in. Schmidt telescope; and $278 \AA / \mathrm{mm}$ with the 48 -in. Schmidt.

The role of the single slit diffraction pattern of the facets of the lamellar grating is different from that for the Wood grating; the facets are here only half a grating groove width-accordingly, diffraction spreading is twice as great. This wider pattern is centered on the zero, rather than on a lateral first order spectrum. Null points of the diffraction pattern fall on all lateral even orders, which should be weak or missing. But the central undispersed image falls on the central diffraction maximum. If interference did not play a contravening role, such an enveloping diffraction pattern would make the relative intensities of the central and symmetrical orders, respectively:

$$
\text { 1: } \frac{4}{\pi^{2}}: 0: \frac{4}{\pi^{2}} \times \frac{1}{3^{2}}: 0: \frac{4}{\pi^{2}} \times \frac{1}{5^{2}}: 0: \text { etc. }
$$

However, for $\lambda_{0}$, the wavelength for which the relative retardation of the rays through the uncoated and quartz-coated strips (of equal area) amounts to a relative phase reversal, the central order should disappear; and its intensity at nearby wavelengths should be weak. Accordingly, in the lamellar grating here, we predict, from the diffraction pattern, that the ratio of the light at $\lambda_{0}$ in one of the first orders, to the total light incident is

$$
\frac{1}{2\left[1+\left(1 / 3^{2}\right)+\left(1 / 5^{2}\right)+\text { etc. }\right]}=\frac{3}{\pi^{2}} \text {. }
$$

This ratio of brightness of the lamellar orders is not as disadvantageous, compared with the brightness by a Wood-type grating, as one might at first conclude: there are information advantages in photographing two spectra, at half exposure, over photographing all the light in one spectrum.

The theory of the lamellar grating has been known for over a century, from Quincke's 1867 paper. ${ }^{1}$ But this type of grating has had little application in the visible spectrum until development of the art of thermal evaporation. However, reflection lamellar gratings have been used effectively in far ir spectroscopy. ${ }^{2}$ And, provided with variable groove depth, they have been employed for Fourier spectroscopy in the far ir where they have many advantages. ${ }^{3}$

The wavelength $\lambda_{0}$ that is missing in the central order, is determined by the thickness of the quartz strips $-t=0.5 \mu$. The relative retardation for normal incidence is

$$
(N-1) t=\frac{1}{2} \lambda_{0}, \text { for } \lambda_{0}=4800 \AA .
$$

And the interference cancellation in the central order is nearly complete for adjacent wavelengths. One can make $\lambda_{0}$ effectively longer by tipping the grating: At incidence angle $\alpha$ (refraction angle $\beta$, in the quartz), the relative retardation is $(N \cos \beta-\cos \alpha) t$. If we differentiate this with respect to $\alpha$,

$$
\lim _{\alpha \rightarrow 0} \frac{\sin \alpha}{N}\left(N-\frac{\cos \alpha}{\cos \beta}\right) \rightarrow \frac{N-1}{N} \alpha .
$$

We see that the retardation increases as $\lambda_{0}$ increases. If the grating is tipped about an axis that is perpendicular to grating grooves, the central image, absent at $\lambda_{0}=4800 \AA$, practically disappears in the visible but the dispersion does not change. Tipped about a parallel axis, the central image again disappears; but the dispersion increases markedly. These results indicate that field corrections will be simpler than with an objective prism.

\section{References}

1. G. Quincke, Pogg. Ann. 132, 364 (1867).

2. R. M. Badger and C. H. Cartwright, Phys. Rev. 33, 692 (1929).

3. G. A. Vanasse and H. Sakai, in Progress in Optics, E. Wolf, Ed. (North-Holland Publishing Company, Amsterdam, 1967), Chap. 7.

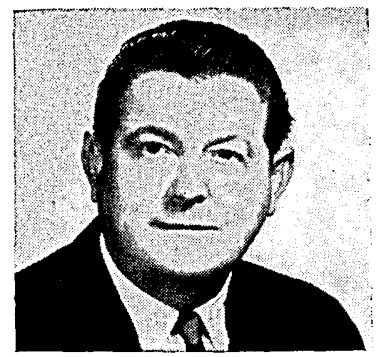

Nelse Einwaechter of the Goerz Optical Company. 